\title{
The relationship between dextranase enzyme in mouthwash and dental caries
}

\author{
Piyachat Tongpong1', Sasithorn Sirilun², Chaiyavat Chaiyasut ${ }^{3}$, \\ Phakkharawat Sittiprapaporn ${ }^{4}$
}

${ }^{1}$ Researcher, Brain Science and Engineering Innovation Research Group, School of Anti-Aging and Regenerative Medicine, Mae Fah Luang University, Bangkok, Thailand, and Department of Anti-Aging Science, School of Anti-Aging and Regenerative Medicine, Mae Fah Luang University, Bangkok, Thailand, ${ }^{2}$ Assistant Professor, ${ }^{3}$ Assistant Professor and Head, Innovation Center for Holistic Health, Nutraceuticals and Cosmeceuticals, Faculty of Pharmacy, Chiang Mai University, Chiang Mai, Thailand, ${ }^{4}$ Assistant Professor and Head, Brain Science and Engineering Innovation Research Group, School of Anti-Aging and Regenerative Medicine, Mae Fah Luang University, Bangkok, Thailand, and Department of Anti-Aging Science, School of Anti-Aging and Regenerative Medicine, Mae Fah Luang University, Bangkok, Thailand

Background: Several studies reported that Thai people have oral health problems as a major problem. Therefore, care and treatment of oral health is an issue to be attentive. At the present, there are many products for oral and dental health care in many forms such as toothpaste, mouthwash, spray, deodorizing and inhibiting bacteria etc. Aims and Objective: This study investigated the effect of mouthwash containing Dextranase enzyme which affected to the changes of dental plaque (Plague Index) in eighteen dental caries volunteers by two mouthwash formulas; Formula I contained only Dextranase enzyme whereas Formula II contained a mixture of Nisin and Dextranase enzymes. Materials and Methods: This study was a single blind randomized clinical trial study. All participants were divided into two equal number of groups received mouthwash containing Dextranase enzyme or mouthwash containing a mixture of Nisin and Dextranase enzyme after using mouthwash. The study allowed participants to use mouthwash continuously for at least four weeks. The participants received oral examination, oral digital imaging and proximal radiographs using Bitewing oral Imaging method, respectively. Results: Both before and after using mouthwash were found to have dental caries. The study found that plague index in participants were decreased. Although the results of this study show that using mouthwash containing Dextranase enzymeeffect to decreased plaque accumulation on teeth volunteers were found to have dental caries but there are other factors besides the plaque on the teeththat causes dental cariese.g. susceptible tooth surface, Acidic product and $\mathrm{pH}$ change. Conclusion: Using mouthwash containing Dextranase enzyme help to reduce plaque formation.

Key words: Mouthwash; Dextranase enzyme; Dental caries lesion
http://nepjol.info/index.php/AJMS DOI: 10.3126/ajms.v11i1.26531 E-ISSN: 2091-0576 P-ISSN: 2467-9100

\section{INTRODUCTION}

Oral health is very important in daily life which in addition to reflecting general physical health. There is still a great deal of communication reflections on personality. Therefore, taking care of oral health and teeth to be healthy is as much as necessary. With other body health Which from the $5^{\text {th }}$ National Dental Health Survey in 2002 by the
Bureau of Dental Health, Department of Health, Ministry of Public Health, Thailand, in the 6 age groups, 3, 5-6, 12 , $15,35-44$ and $60-74$ years, it was found that young children had high prevalence and severity of caries. By finding the population of dental caries in rural areas more than in urban areas which oral health problems in dental caries often occur in children. While adults and the elderly have dental caries and periodontal problems. ${ }^{1}$ 
Several studies reported that Thai people have oral health problems as a major problem. Therefore, care and treatment of oral health is an issue to be attentive. At the present, there are many products for oral and dental health care in many forms such as toothpaste, mouthwash, spray, deodorizing and inhibiting bacteria etc.The application of products derived from beneficial bacteria such as Nisin which is a metabolite producing from lactic acid bacteria, a safe bacterium. Nisin is used as a biological preservative. Many European countries reported that inhibiting pathogenic microorganisms can be detected. ${ }^{2} \mathrm{It}$ also reports that Nisin can inhibit the biofilm formation of S. mutans bacteria because Nisin is a bacteriocin that has a broad inhibitory effect and safe. ${ }^{3}$

In addition, Khalikova et al. ${ }^{4}$ reported dextranase enzyme has ability to cut $\alpha-1,6$ glucosidic linkages of dextran, which is the structure of bacteria biofilm adhesion of dental caries and periodontal disease. Reduction of biofilm stains resulted in reduction dental caries, periodontal disease, and inflammation. After Block et $\mathrm{al}^{5}$ conducted a hamster trial for 35 days, Schachtele et al. ${ }^{6}$ found that Dextranase had an effect on the formation of S. mutans in the oral cavity. Removal of oral biofilm stains is an interesting mechanism.

Dextranase is an enzyme that catalyzes the endohydrolysis of $\alpha$-(1-6)- D-glycoside linkages in dextran. ${ }^{7}$ The dextran plays a critical role in dental plaque, which is one of the key players involved in the development of some common oral diseases like dental caries. So, several researchers investigating dextran-hydrolyzing enzymes for the clinical and industrial applications. ${ }^{8}$

The oral and dental health care by Nisin and dextranase enzymes is also a new story for Thai people. Therefore, it is interesting another way to reduce biofilm stains and reduce the amount of dental caries microorganisms which are oral health problems.In order to eliminate existing problems and prevent future problems, this will develop Thai products to help reduce dental caries problems of the Thai population in which the oral health is very important in daily life in addition to reflecting general physical health. There is still a great deal of communication and personality as well.

This study aims to study the effect of mouthwash containing dextranase enzyme and a mixture of Nisin and dextranase enzymes to reduce the indicator associated with dental caries which is the reduction of biofilm stains S. mutans microbial caries. In the sample with dental caries will help to reduce the occurrence of dental caries in the same area and prevent new tooth cavity and the original dental caries will not spread violently while waiting a dental treatment appointment.

\section{MATERIALS AND METHODS}

\section{Population and sample used in the study}

Participants with dental caries on the teeth at the dental clinic of the day Pak Nam Market Branch, Samut Prakan Province, Samut Prakan Province during December 2018 to January 2019. Sample size calculation of dental caries group using STATA program which based on previous research studies. For the expected value, the S. mutans microbial index calculated as seven participants per group and 30\% dropout rate. Therefore, nine participants per group were participated in this study. Thus, there were 18 participants totally involed in this study.

\section{Bitewing oral radiography}

This type of radiation is a radiographic image that can help diagnose the disease very well because it can show the true dimension of the teeth because of the slightest magnification or contraction of radiation. Many pathologic which could not be seen from the radiograph around the root (Periapical radiographic, $\mathrm{Pa}$ ) should clearly from this radiographic image such as initial stage dental caries or enamel caries, the depth of the tooth restoration or dental caries cavities, the relationship between recurrent caries, depth of tooth decay and pulp, the defect of marginal crown restoration, respectively. The examination of the bitewing radiographic was the most effective way to diagnose dental caries at proximal area.

\section{Periodontal examination method}

Periodontal Screening and Recording (PSR) Scores were assessments of periodontal conditions using the Periodontal probe which had a black band at a distance of 3.5-5.5 mm. This study used a probe to inspect every tooth at least 6 positions per tooth, including the side near the middle of the cheek (mesio-buccal: MB), cheek midpoint (mid-buccal) on the middle of the cheek (disto-buccal: DB) near the middle of the tongue (mesio-lingual: ML) midpoint on the tongue (mid-lingual) and the middle side of the tongue (disto-lingual: DL), respectively. Gently walked the probe along the tooth and recorded the highest score in each sextant into the table that represented the sextant. As shown in the picture, Periodontal disease was diagnosed following principlesfrom the table.

\section{Guidelines for measuring plaque index}

Dye plaque of all teeth with erythrosine solution. Throughout the tooth surface after 15 seconds, let participants rinsed with water. Check plaque by detected a clear red line aligned with the gum line on the tooth surface.Recording 4 positions: buccal, lingual, mesial and distal side Only 6 representative teeth are 16, 21, 26, 36, 41,46 . In the event that the representative tooth was not fully eruption, crown restorations or retained root teeth 
would not record plaque but consider recording adjacent teeth instead.

Assessing score of plaque in the mesial and distal sides of the buccal and lingual surfaces. Recording the higher scorefor example, if distobuccal side score is 2 , distolingual side score is 3 , and score of plaque will recorded in 3 . The plaque index used to improve from Bay and Ainamo Visible Index. With the criteria of the index as follows:

$0=$ No plaque

1 = Slightly visible plaque as seen as a spot ofdye on cervical area.

$2=$ Visible plaque band on cervical area notmore than $1 / 3$ of tooth clinical crown.

$3=$ There are more than $1 / 3$ of plaque coveredin the tooth clinical crown.

Recorded values to calculate the average plaque index of volunteers from formula.

Plaque Index $(\mathrm{PI})=$ The sum of the plaque index

\section{Plaque Index $(\mathrm{PI})=\underline{\text { The sum of the plaque index }}$}

\section{Number of positions checked (or $=24 *$ )}

*Note: Examining 6 teeth, each position is 4 positions. Therefore, the number of positions examined $=24$

Irritation assessment of formulas in participants by patch test

Cleaning the left arm area of participants with distilled water (DI Water). Position measurement for irritation test by measuring 25 centimeters from the middle finger. Draw a box for applying $2 \times 2 \mathrm{~cm}$ products $(1 \mathrm{~cm}$ apart). Apply 200 micrograms of products that need to be tested throughout the area. (Apply the product randomly) by applying clockwise 5 times. Leave to dry for 1 hour. After 1 hour, read the results and interpreted the results based on the International Contact Dermatitis Research Group (ISDRG). Participants who did not have any irritating reactions could join the project. Recommendations to all participants included: (1) participants should be careful not to get wet skin or get moisture, (2) during test should refrain from activities that caused excessive sweating such as exercise, (3) during test might be slight itching or irritation from chemicals caused reactions small allergic rashes at test area.

\section{Guidelines for proper mouthwash}

Rinse the mouthwash in a $20 \mathrm{ml}$ container (4 teaspoons). Pour the mouth into the mouth without having to dilute the water into the mouthwash.Scour the mouthwash for
30 seconds (count 1-30 in mind). Rinse the liquid in the sink. To increase the effectiveness of mouthwash, it should not rinse water after used.

\section{The research instrumentt}

Participant Records consists of personal information such as name-surname, sex, age, congenital disease, medication received prior to study which was no more than 3 months, allergy information, smoking and alcohol information, how to clean the mouth/oral care, dental treatment history and contact dentist telephone number.

Mouthwash:

Formula 1: mouthwash containing Dextranase enzyme.

Formula 2: Mouthwash containing Nisin and Dextranase enzyme. $6 \%$ erythrosine solution is the color used to stain the plaque of the teeth manufactured by the Faculty of Dentistry Mahidol University.

The periodontal probe which has a black band at a distance of $3.5-5.5 \mathrm{~mm}$.

Digital camera with Huawei mobile phone series P20 Pro (Huawei) manufactured by Huawei Technologies Co., Ltd., used for participants intraoral picture before project began and after staining the plaque both before and after using mouthwash.

The form used to evaluate and record results of the study including (1) dental examination record for recording the plaque index and periodontal conditions, (2) participant's journal contains practical guidelines during the research. Receiving test products and use of mouthwash formulas. Daily recording sampling. Instructions for mouthwash, (3) sensory evaluation form.

\begin{tabular}{ll} 
Table 1: Criteria periodontal diagnosis & \\
\hline Code & Diagnosis \\
\hline$P S R=0$ & Normal \\
$P S R=1$ & Gingivitis \\
$P S R=2$ & Periodontitis \\
$P S R=3$ (pocket $3.5-5.5 \mathrm{~mm})$. & \\
$P S R=4$ (pocket $>5.5 \mathrm{~mm})$. & \\
Code $=^{*}$ mobility, furcation involvement & \\
\hline
\end{tabular}

Table 2: Comparison Plaque Index in volunteers before and after used mouthwash

\begin{tabular}{lccc} 
Volunteer group & \multicolumn{2}{c}{$\begin{array}{c}\text { Median (Quartile range) } \\
\text { (minimum - maximum) }\end{array}$} & p-value \\
\cline { 2 - 3 } & Before & After & \\
\hline Dental caries volunteer & $2.25(1.08)$ & $1.50(0.67)$ & 0.0280 \\
mouth wash formula 1 & $(1.42-2.67)$ & $(1.00-2.00)$ & \multirow{2}{*}{0.0277} \\
Dental caries volunteer & $2.085(0.67)$ & $1.75(0.5)$ & \\
mouth wash formula 2 & $(1.58-3.00)$ & $(1.25-2.33)$ & \\
\hline
\end{tabular}

Asian Journal of Medical Sciences | Jan-Feb 2020 | Vol 11 | Issue 1 


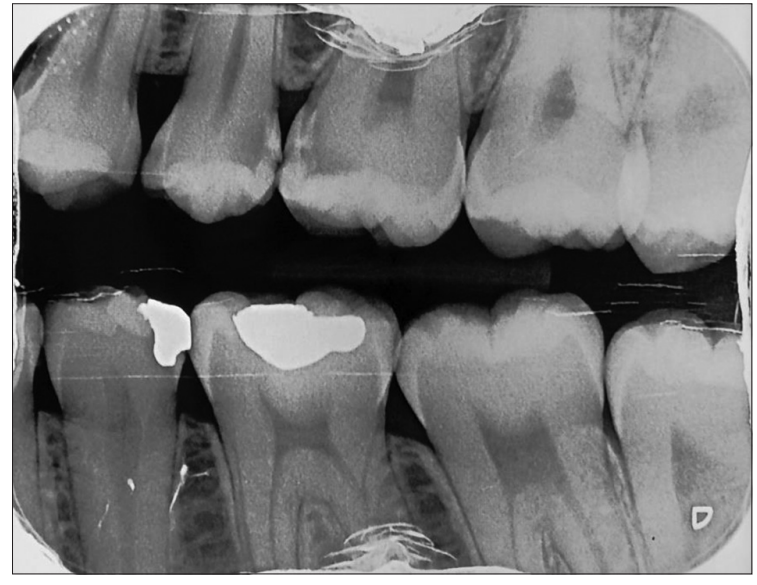

Figure 1: Bite wing film shown proximal caries

Research method

Week 0 (baseline): Intraoral x ray Bit wing film. Took intraoral pictures with digital cameras before dyeing plaque with Erythrosine solution. Dye plaque with Erythrosine solution and took intraoral pictures after dyeing.Dentist recorded and analyzed Plaque index.

Week 4: Evaluation step after volunteers used mouthwash for at least 4 weeks doing the same procedure as the 0 week Except the satisfaction assessment including (1) intraoral $\mathrm{x}$ ray Bit wing film, (2) took intraoral pictures with digital cameras before dyeing plaque with Erythrosine solution, (3) dye plaque with Erythrosine solution and take intraoral pictures after dyeing, and (4) satisfaction assessment.

\section{RESULTS AND DISCUSSION}

Selection volunteers with dental caries lesion Visual examination and Intraoral x ray Bite wing film.

\section{Irritation assessment}

Assessment allergic and irritant test results by testing in 18 participants whom tested the formula 1 (F1) mouthwash and formula 2 (F2) mouthwash showing visual assessment results by tracking symptoms. Desirable that may occur closely study of skin irritation assessment of mouthwash formulations containing dextranase formula 1 (F1) and mouthwash containing dextranase and nisin formula 2 (F2). All 18 participants using patch test found not cause allergic or irritant reactions with 5 levels assessment criteria with photographic at the test area both before and after applying the product as shown below picture.

The effect of formulations containing nisin and/or dextranase enzymes on plaque index in volunteer with dental caries lesion

Evaluating the effect of mouthwash on Plaque index at 0 and 4 weeks by using Wilcoxon signed-rank test statistics as shown in the Tables 1 and 2 .
The results showed that Plaque index (PI) of both groups decreased significantly. The mouthwash containing dextranase (formula 1) and mouthwash containing dextranase and nisin (formula 2) affected to Plaque index (PI) of volunteers.

The study found that plague index decreased after using mouthwash continuously for at least four weeks. The effect of mouthwash containing Dextranase enzyme bothformulas which Formula I contained only Dextranase enzyme whereas Formula II contained a mixture of Nisin and Dextranase enzymes affected to the changes of dental plaque (Plague Index) in eighteen dental caries volunteers by assessmentwith intraoral picture. Although the results of this study show that using mouthwash containing Dextranase enzyme effect to decreased plaque accumulation on teeth volunteers were found to have dental caries but there are other factors besides the plaque on the teeththat causes dental caries e.g. susceptible tooth surface, Acidic product and pH change (Figure 1).

\section{CONCLUSION}

Therefore, in addition to just using mouthwash containing Dextranase enzyme that reduces plaque formation, there should be products that reduces the factors causing dental caries as mentioned and limitations of this study a smaller number of dental caries volunteers they cannot represented in a large population. Otherwise, just brush your teeth properly and add mouthwash to reduce plaque formation. Where brushing is not complete should be the best way to prevent tooth decay which still requires further study in the future.

\section{ACKNOWLEDGEMENT}

The authors would like to acknowledge the Thailand Research Fund (TRF), Supporting Research Funds for Industries (SURF) (Grant no. RDG58I0002), for the support. The authors gratefully acknowledge the Chiang Mai University grant (CMU-grant) for the support. Authors also gratefully acknowledge Brain Science and Engineering Innovation Research Group, Mae Fah Luang University grant (2019) (MFU-grant no. 611U109005) and 2020, the supports and necessary provision.

\section{REFERENCES}

1 Number of Donors, Dental Public Health Division, Department of Health, Ministry of Public Health, "Report of the dental condition survey $5^{\text {th }}$ National Health $2000-2001,2002$.

2. Tong Z, Zhou L, Jiang W, Kuang R, Li J, Tao R, et al. An in vitro synergetic evaluation of the use of nisin and sodium fluoride or 
chlorhexidine against Streptococcus mutans. Peptides 2011; 32(10): 2021-2026.

3. Pringsulaka O. Bacteriocin generated from lactic acid bacteria (Bacteriocins of Lactic Acid Bacteria). Science Journal Srinakharinwirot University 2007; 23(2): 148-149.

4. Khalikova E, Susi P and Korpela T. Microbial Dextran-Hydrolyzing Enzymes: Fundamentals and Applications. Microbiology and Molecular Biology Reviews 2005; 69(2): 306-325.

5. Block PL, Dooley CL and Howe EE. The retardation of spontaneous periodontal disease and the prevention of caries in hamsters with dextranase. Journal of Periodontology 1969;
40(2): 105-110.

6. Schachtele CF, Staat R and Harlander SK. Dextranases from oral bacteria: inhibition of water-insoluble glucan production and adherence to smooth surfaces by Streptococcus mutans. Infection and Immunity 1975; 12(2): 309-317.

7. Wang DL, Lu MS, Wang SJ, Jiao YL, Li WJ, Zhu Q, et al. Purification and characterization of a novel marine Arthrobacter oxydans KQ11 dextranase. Carbohydr Polym 2014; 106: 71-76.

8. Khalikova E, Susi P and Korpela T. Microbial dextran hydrolyzing enzymes: fundamentals and applications. Microbiol Mol Biol Rev $2005 ; 69(2): 306-325$.

\section{Authors Contribution:}

PT- Concept and design of the study, statistically analyzed and interpreted, manuscript preparation; SS- Concept and design of the study, statistically analyzed and interpreted; CC-Concept and design of the study, statistically analyzed and interpreted; PS- Concept and design of the study, statistically analyzed and interpreted, manuscript preparation, critical revision of the manuscript.

Work attributed to:

Brain Science and Engineering Innovation Research Group, School of Anti-Aging and Regenerative Medicine, Mae Fah Luang University, Thailand. Innovation Center for Holistic Health, Nutraceuticals and Cosmeceuticals, Faculty of Pharmacy, Chiang Mai University, Thailand.

\section{Orcid ID:}

Mrs. Piyachat Tongpong - (10 https://orcid.org/0000-0003-2630-809X

Dr. Sasithorn Sirilun - id https://orcid.org/0000-0002-1508-2358

Dr. Chaiyavat Chaiyasut - (10) https://orcid.org/0000-0002-1633-2419

Dr. Phakkharawat Sittiprapaporn - (i) https://orcid.org/0000-0002-4103-9396

Source of Support: Thailand Research Fund (TRF), Supporting Research Funds for Industries (SURF) (Grant no. RDG58I0002); Chiang Mai University grant (CMU-grant), Thailand; Brain Science and Engineering Innovation Research Group, Mae Fah Luang University grant (2019) (MFU-grant no. 611U109005) and 2020, Thailand. Conflict of Interest: None declared. 